\title{
Industrial Research and Development with Synchrotron Radiation
}

\author{
Katja Kroschewski ${ }^{1, a}$, Adam Webb ${ }^{2, b}$,Thomas Wroblewski ${ }^{2, \mathrm{c}}$ and \\ Karsten Wurr ${ }^{1, d}$ \\ ${ }^{1}$ Servicegroup Industry, TT, DESY, Notkestr. 85, D-22607 Hamburg, Germany \\ ${ }^{2}$ Servicegroup Industry, HASYLAB, DESY, Notkestr. 85, D-22607 Hamburg, Germany \\ akatja.kroschewski@desy.de, badam.webb@desy.de, 'thomas.wroblewski@desy.de, \\ dkarsten.wurr@desy.de
}

Keywords: Industry, Synchrotron radiation, Applied research

\begin{abstract}
The outstanding properties of synchrotron radiation (SR) allow manifold investigations of materials and processes which are not possible with conventional X-ray sources. Its high brightness allows extremely precise or time/spatial resolved measurements. In combination with its high collimation extremely high angular resolution is achievable. The white spectrum of SR allows tuning of the wavelength for spectroscopic applications or optimization according to the requirements of the experiment.
\end{abstract}

\section{Introduction}

Material properties are not only determined by their composition but also by the various treatments (thermal, mechanical, chemical, etc.) during production and use (strain, wear, etc.). Manifold SR based techniques enable not only the investigation of these properties but also the observation of dynamic processes occurring during the lifetime of the product. For this purpose conventional methods as they are also available using X-ray tubes may be applied. Examples are radiographic methods, diffraction and fluorescence analysis. SR experiments, however, go beyond the possibilities of these conventional techniques. Tunability of the wavelength allows contrast variation and element specific radiography and tomography[1]. The high collimation can be used for contrast enhancement (Diffraction enhanced imaging [2]). Furthermore, phase contrast imaging techniques may also be used [3].

In diffraction the high intensity allows the structure determination of proteins before they are damaged by secondary processes following the irradiation. Parallel beam geometry in polycrystalline diffraction is aberration free and thus allows the investigation of objects even if they deform or move [4].

Also in fluorescence analysis the properties of SR (which is polarized) allow the detection of minor traces down to the fg level [5] or the non destructive investigations of inclusions (in confocal geometry) [6].

The scope of this paper is not to describe these extensions of conventional techniques but to present novel developments in the investigation of materials which are only possible with SR.

\section{Spectroscopy}

X-ray absorption spectroscopy (XAS) requires a tuneable source with high intensity and good collimation and is thus only possible with SR. This is particularly the case in the investigation of dynamic processes. In contrast to diffraction, crystalline specimens are not necessary but rather amorphous or nano-crystalline substrates may also be investigated. This makes XAS an ideal tool for the investigation of heterogeneous catalysis in which the catalysts have a large surface to volume 
ratio and may undergo structural changes. The ultimate goal is to understand the details of the active site under operating conditions. This includes not only the atomic structure, local environment and oxidation state of the key elements but also the particle size, shape and dynamics of the active species. XAS is used in addition to methods such as X-ray diffraction and TEM. One powerful technique is to make in situ XAS measurements in addition to monitoring the reaction process using an analytical tool such as a mass spectrometer or GC $[7,8]$. Capillary reactors and reactor cells have been developed by Haldor Topsøe A/S [8,9]. Examples of industrial uses include methanol synthesis [9], high temperature shift catalysts [7] and steam-reforming catalysts. These investigations led to deeper understanding of the underlying processes and thus allowed improvement of the catalysts. It could be shown, that in methanol synthesis metallic $\mathrm{Cu}$ nanoparticles form from the $\mathrm{Cu} / \mathrm{ZnO}$-based catalyst which are the active component. The morphology of these particles changes according to the conditions in the reactor. Based on this knowledge the synthesis can be optimized.

Temporal techniques such as QEXAFS (Quick EXAFS) have been developed to follow structural changes of the catalyst in situ. Alternatively the time scale may be transformed to a spatial scale by imaging of the reaction front. Tuning the energy of the incident X-rays can be used for 2D mapping inside a catalytic micro-reactor during operation [7,11]. Careful selection of the energy was used to map the distribution of oxidized/reduced forms of $\mathrm{Rh}$ and to visualize the reaction front in the reactor [12] during partial oxidation of methane. It was observed that the reaction rate was higher in the middle of the reactor which is consistent with a radial temperature gradient from the centre outward. The profile also supports speculation that methane combustion occurs in the first part (at low temperature) followed by methane reforming in the second part.

Low temperature fuel cells have been investigated using both transmission and fluorescence geometries during cell operation [13]. XAS is used to investigate changes in oxidation state, particle size, alloying, adsorbate coverage etc. under a variety of atmospheric and temperature conditions.[14,15] Structural changes at the anode and cathode with potential are of particular interest. XAS may be further complemented by other techniques like small angle scattering or powder diffraction.

\section{Diffraction Imaging}

X-ray diffraction imaging is a novel technique applying an array of parallel tubes in front of a position sensitive detector [16]. These tubes suppress crossfire of radiation diffracted from different parts of a polycrystalline specimen thus allowing the imaging of a large area in a single shot. An instrument dedicated to this method has been realized at HASYLAB beamline G3[17]. It uses a micro channel plate (MCP) as a collimator array in front of a CCD camera. This MCP/CCD arrangement is mounted to the detector arm of a four circle diffractometer and can be moved radially to optimize the sample to detector distance according to the sample and its environment (the spatial resolution is given by the angular acceptance of the MCP times the sample to detector distance and should thus be minimized). A $\theta-2 \theta$-scan with this instrument yields a series of images displaying the spatial resolved intensity diffracted in the direction of the tubes of the MCP. These data maybe rearranged yielding up to one million diffractograms (one for each pixel of the CCD) corresponding to the different sample regions. To handle this large amount of data various techniques for data reduction and processing have been developed or adapted from other disciplines (for example, data sets from remote sensing have a similar structure - i.e. images from a single scene but at different wavelengths). The aim of these procedures is to sort the data according to their diffractograms into a few classes and to obtain maps of their spatial distribution.

Mapping of composition and strain Engineering materials often show inhomogenities, for example in the vicinity of welds. The formation of intermetallic phases is of special interest in dissimilar welding. A rotational friction welded sample of A17020/Ti6Al4V, produced at GEA Tuchenhagen $\mathrm{GmbH}$, Büchen, Germany, was mounted such that the weld was aligned parallel to the 
diffraction plain [18]. The structural variation was, therefore, only perpendicular to this plane allowing integration in the direction of the weld leading to a significant data reduction. Fig. 1 shows the diffracted intensity in the vicinity of the weld as function of $2 \theta$ and the distance from the weld. The intensity on the Ti side is generally higher due to the Ti fluorescence. The peak shift is due to a continuous increase of the Al concentration $(\sim 200-100 \mu \mathrm{m})$ finally resulting in the formation of $\mathrm{Ti}_{3} \mathrm{Al}(\sim 100-0 \mu \mathrm{m})$. Stress was determined in another investigation on the Al-side of the weld applying the $\sin ^{2} \psi$ method. Tensile strain was found in the heat affected zone, reaching its maximum of $90 \mathrm{MPa}$ at distances of roughly $2 \mathrm{~mm}$ from the weld[19]. Further investigations are planned for the Ti-side of the material.

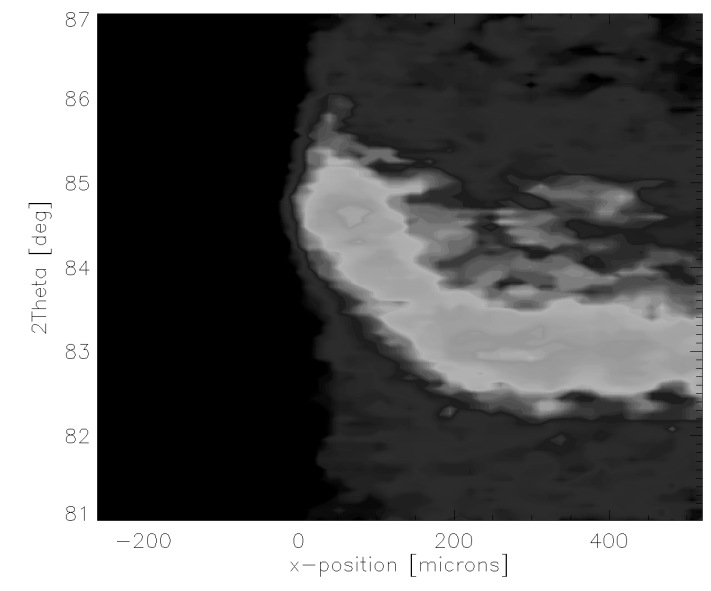

Fig. 1: Intensity as function of $2 \theta$ and distance from a dissimilar weld.

The Ti side generally shows higher intensity due to the $\mathrm{Ti}$ fluorescence. In the region from 0 to $100 \mu \mathrm{m} \mathrm{Ti}_{3} \mathrm{Al}$ has formed while the $\mathrm{Al}$ concentration gradually decreases from 100 to $200 \mu \mathrm{m}$.

Structural changes also occur during use of materials. An example is the white etching layer formed on rails due to the repeated load by fast and/or heavy trains. This martensitic phase is randomly distributed on the rail surface. Furthermore, its reflections exhibit a strong overlap with the underlying ferrite and the very fine grained structure leads to peak broadening. Additionally the intensity at the energy of $6.5 \mathrm{keV}$ used to suppress the Fe fluorescence was rather low. All this led to rather noisy images requiring more sophisticated methods for data reduction [20]. A principal component analysis was applied to a set of 101 exposures around the ferrite 110 reflection. The result of such a transformation is a series of so called eigenimages which can be sorted according to their signal to noise ratio. While the first eigenimage represents the average intensity the second eigenimage (Fig.2) clearly shows the spatial distribution of the two phases. By applying a simple threshold the regions containing the two different phases could be selected and their structural properties extracted.

The fact that the entire sample is investigated simultaneously allows the investigation of dynamic processes of which no prior knowledge where they occur is available. Recrystallization is such a process and can be monitored by adjusting the MCP/CCD combination to one reflection (preferentially to the strongest peak according to recrystallization texture). The position of recrystallized grains can be determined from the finite (or intermediate) images and their history can be traced back to the very early stages of nucleation. Investigations of grain evolution showed a stochastic behaviour which may be described by the model of self-organized criticality [21]. In the process of aluminium recrystallization, shrinkage of grains which nucleated very early and than grew to a significant size could be observed [22]. 


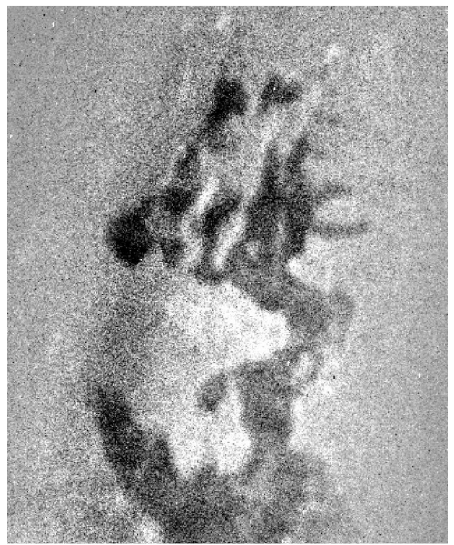

Fig. 2: Second eigenimage resulting from a principal components transformation of 101 exposures from a scan over the Fe 110-reflection. Dark areas correspond to the white etching layer while the untransformed ferrite is located in the bright regions. (Sample provided by the Deutsche Bahn AG)

\section{Interaction of Industry and Science}

Fundamental research aims to realize model systems with well defined properties for investigation. Such systems are far from those arising in industrial application. It is therefore challenging to modify existing techniques or even develop novel methods for industrial research. Examples include the development of various sample environments like the above mentioned reaction cells for XAFS but also the implementation of data evaluation procedures to handle the huge amount of data arising from the various imaging techniques. The combination of simultaneous measurement (imaging) with joint data processing is essential to efficiently handle large sample quantities as they arise, for example, in combinatorial synthesis. A clear example for the development of a new technique for the mutual benefit of both industry and research lab is depicted by the investigation of rotating parts for the automotive industry [23]. These parts are subject to friction and wear, resulting in residual stress leading finally to failure. The examined sample consisted of a cylinder with grooves due to the treatment with sand paper. To determine the residual stress distribution, position resolved measurements had to be performed under variation of the angle of incidence $\left(\sin ^{2} \psi\right.$ method). First the cylindrical shape of the specimen and its coarse grains seemed to be a problem, because of the definition of the surface normal and the need to vary the sample orientation to avoid artefacts due to the orientation of single grains, but during discussion it turned out that just this variation could be used to significantly reduce the measuring time. The grooves follow the circumference of the rod. Therefore, the structural variation is only in the direction of the axis. The variation of the surface normal could thus be used for the variation of $\psi$. Furthermore the sample was rotated around its axis thus overcoming the problem of graininess. With the wavelength chosen thus that the Bragg angle of the investigated reflection was $45^{\circ}$, the measurement $\left(-45^{\circ}<\psi<45^{\circ}\right)$ could be performed in a single $2 \theta$ scan yielding a series of images with the sample axis (strain variation) in one direction and the $\psi$ related information perpendicular.

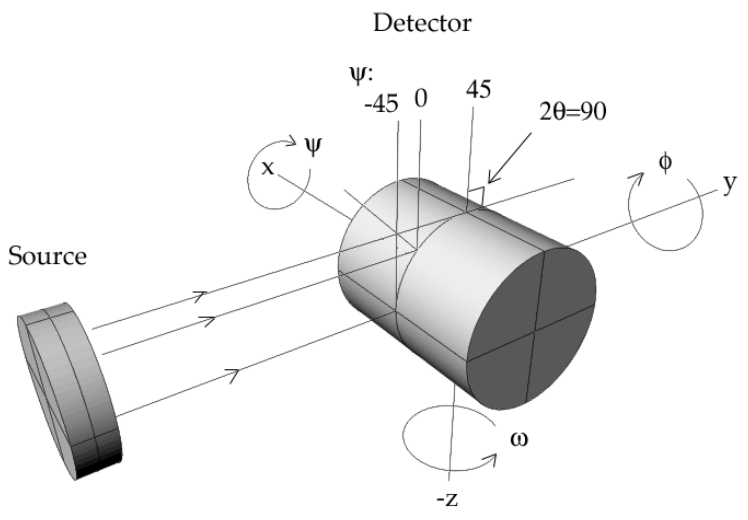

Fig. 3: Experimental set-up for the determination of residual stress in a cylindrical sample. During the measurement the sample is continuously rotated around its axis to overcome the problem of graininess. The variation in $\psi$ is projected onto the $\mathrm{y}$ axis of the detector. (Courtesy by S.K. Ås) 


\section{Access for Industry}

In an industrial setting usually time is much more at a premium than money, thus industry often needs rapid access to beam time at a synchrotron. Also, in many cases companies do not want to publish their research results or even the subject of their research, but rather keep it confidential to retain their competitive edge. Therefore they are not interested in going through the usual peer review process at publicly funded research infrastructures. DESY offers such rapid access for industry to synchrotron radiation, where the industrial users can bypass the peer review process for a cost compensation that varies according to the nature of the research carried out, i.e. published or confidential. Industrial beam time is provided either via short term projects or the HASYLAB cooperation model, where users enter into a 3 year contract with DESY for a fixed amount of money against which they can use beam time. Both variants of access can be booked with or without full or supporting service [24]. Industrial Users at DESY receive assistance from HASYLAB's Service Group Industry, which also handles organisational matters and advises customers on the realisation and possibilities of measurement projects. If suitable this is done in collaboration with one of DESY's onsite partners: GKSS, EMBL or MPG. These groups operate their own beamlines and instrumentation (GKSS: materials science, EMBL and MPG: protein crystallography) at the storage ring DORIS, a second generation source well-suited for high flux applications.

In the fall of 2009 DORIS will be supplemented by PETRA III, which will be one of the most brilliant storage ring-based sources of X-ray radiation in the world. As the most powerful light source of its kind, it will offer scientists and industry alike outstanding experimental opportunities with X-rays of an exceptionally high brilliance and will therefore be ideally suited for investigations on the micron and submicron scale. In particular, this will benefit researchers investigating very small samples or those requiring tightly collimated and very short-wavelength X-rays for their experiments. A similar industrial access model for rapid access is planned for PETRA III.

\section{Conclusion}

Synchrotron radiation, with its high brightness and collimation, opens diverse possibilities for investigating materials and processes in an extremely precise and time/spatial resolved manner.

Among the measurement techniques are X-ray absorption spectroscopy, Quick EXAFS and sophisticated imaging techniques to analyse catalysts in situ, which are of interest to companies producing catalysts for the chemical and automotive industry.

Another method provided by SR is X-ray diffraction imaging used for the mapping of composition and strain, for example in the vicinity of welds and structural changes that can occur during production, machining and use of the material.

Currently, DORIS III, the $2^{\text {nd }}$ generation light source at DESY, and from the end of 2009 onwards, PETRA III, the $3^{\text {rd }}$ generation light source, offer scientific and likewise industrial users a comprehensive set of tools to investigate materials and processes with advanced methods and novel techniques.

\section{References}

[1] F. Beckmann et al., Adv. Eng. Mater. Vol. 9 (2007), p. 939

[2] A. Wagner et al., Phys. Med. Bio. Vol. 51 (2006), p. 1313

[3] U. Bonse, F. Busch, Progress in Biophysics and Molecular Biology Vol. 65 (1996) p.133

[4] J. Ihringer, T. Wroblewski, A. Küster, K. Maichle, Appl. Cryst. Vol. 21 (1988), p. 972 
[5] C. Streli et al., Spectrochimica Acta Vol. B61 (2006), p. 1129

[6] K. Janssens, K. Proost and G. Falkenberg, Spectrochimica Acta Vol. A59 (2004), p. 1637

[7] B.S. Clausen et al., J. Catal. Vol. 132 (1991), p. 524

[8] T.Ressler et al., Physica Scripta Vol. T1 15 (2005), p. 66

[9] J.W. Andreasen et al., J. Appl. Cryst. Vol. 36 (2003) , p. 812

[10] B.S. Clausen, H. Topsøe, Catal. Today Vol. 9 (1991), p. 189

[11] J.-D. Grunwaldt et al., Phys. Chem. Chem. Phys. Vol. 6 (2004), p. 3037

[12] J.-D. Grunwaldt, et al., J. Phys. Chem. Vol. B 110 (2006), p. 8674

[13] C. Roth et al., Adv. Eng. Mat. Vol. 7 (2005), p. 952

[14] B.M. Weckhuysen, In-situ Spectroscpy of Catalysts, (American Scientific Publishers, 2004)

[15] C.Roth et al., Appl Catalysis Vol. A319 (2007), p. 81

[16] T. Wroblewski, et al., Rev. Sci. Instrum. Vol. 66 (1995), p. 3560

[17] T. Wroblewski, et al., Nucl. Instrum. Meth. Vol. A428 (1999), p. 570

[18] H.-G. Brokmeier, et al., in Aluminium Alloys Vol.1, edited by J. Hirsch, B. Skrotzki, G. Gottstein, Wiley-VCH, Weinheim, Germany (2008), p. 947

[19] A.Rothkirch, S. Lenser, Mat. Sci. Forum Vol. 571-572 (2008), p. 237

[20] T. Wroblewski, E. Wild. T. Poeste, A. Pyzalla, J. Mat. Sci. Lett. Vol. 19 (2000), p. 975

[21] T. Wroblewski, Z. Metallkd. Vol. 93 (2002), p. 1228

[22] T. Wroblewski, A. Buffet, in Fundamentals of Deformation and Annealing, edited by P.B. Pragnel and P.S. Bate, Trans Tech Publications LTD, Zurich, Switzerland (2007), p. 631

[23] S.K. Ås, Doctoral thesis at NTNU (2006:25), Trondheim, Norway

[24] Information on http://hasylab.desy.de/user_info/industrial_users/index_eng.html 